\title{
Assessment of the willingness of households to pay for clean water use in Cang Long district, Tra Vinh province
}

\author{
Dung C. Le ${ }^{1}, \&$ Tri D. Pham ${ }^{2}$ \\ ${ }^{1}$ Mekong Delta Development Research Institute, Can Tho University, Can Tho, Vietnam \\ ${ }^{2}$ Tra Vinh Department of Agriculture and Rural Development, Tra Vinh, Vietnam
}

\begin{abstract}
ARTICLE INFO
Research Paper

Received: December 20, 2018

Revised: January 11, 2019

Accepted: January 15, 2019

Keywords

Cang Long district

Clean water in rural area

Contingent valuation method

Estimate

Willingness
\end{abstract}

\section{${ }^{*}$ Corresponding author}

Le Canh Dung

Email: lcdung@ctu.edu.vn

\begin{abstract}
This study aimed to estimate the willingness of households to pay for domestic clean water use against its price in rural areas of Cang Long district, Tra Vinh province. This research also identified the possibility and factors affecting the willingness to pay for a given price of 6,500 VND or above per $\mathrm{m}^{3}$ of clean water. A contingent valuation method with multiple prices (bidding game) ranging from VND 5,000 to VND 9,000 per $\mathrm{m}^{3}$ of clean water was applied to interview 120 households in Cang Long district. Results showed that the mean willingness to pay was VND 6,200 for each $\mathrm{m}^{3}$ of clean water used. The income positively affected the willingness to pay against the price of $6,500 \mathrm{VND}$ or above whereas the household size had a negative impact on this parameter. It is suggested that the service provider should consider to extent the minimum volume of water used up to $10 \mathrm{~m}^{3} /$ month instead of $4 \mathrm{~m}^{3} /$ month in accordance with the minimum price of $5,700 \mathrm{VND}$ per $\mathrm{m}^{3}$ of water used.
\end{abstract}

Cited as: Le, D. C., \& Pham, T. D. (2019). Assessment of the willingness of households to pay for clean water use in Cang Long district, Tra Vinh province. The Journal of Agriculture and Development 18(2), 12-18. 


\title{
Đánh giá mức độ sẵn lòng trả cho sử dụng nước sạch của người dân huyện Càng Long, tỉnh Trà Vinh
}

\author{
Lê Cảnh Dũng ${ }^{1 *}$ \& Phạm Đức Tri ${ }^{2}$ \\ ${ }^{1}$ Viện Nghiên Cứu Phát Triển Đồng Bằng Sông Cửu Long, Trường Đại Học Cần Thơ, Cần Thơ \\ ${ }^{2}$ Chi Cục Phát Triển Nông Thôn Trà Vinh, Trà Vinh
}

\section{THÔNG TIN BÀI BÁO \\ Bài báo khoa học \\ Ngày nhận: 20/12/2018 \\ Ngày chỉnh sửa: 11/01/2019 \\ Ngày chấp nhận: 15/01/2019}

Từ khóa

Đánh giá ngẫu nhiên

Huyện Càng Long

Nước sạch nông thôn

Sự sẵn lòng trả

Ước lượng

* Tác giả liên hệ

Lê Cảnh Dũng

Email: lcdung@ctu.edu.vn

\section{TÓM TẮT}

Nghiên cứu này nhằm ước lượng mức sẵn lòng trả cho mỗi $\mathrm{m}^{3}$ nước sạch của hộ dân ở khu vực nông thôn huyện Càng Long, tỉnh Trà Vinh nơi sắp có nước sạch phục vụ sinh hoạt. Đồng thời nghiên cứu cũng xác định xác suất và các yếu tố ảnh hưởng đến mức sã̃n lòng trả với một mức giá bằng hay cao hơn 6.500 đồng cho mỗi $\mathrm{m}^{3}$ nước sạch. Phương pháp đánh giá ngẫu nhiên (Contingent valuation method) theo thể thức nhiều mức giá (bidding game) từ 5.000 đồng đến 9.000 đồng cho mỗi $\mathrm{m}^{3}$ nước sạch được sử dụng để phỏng vấn đối với 120 hộ dân ở huyện Càng Long. Kết quả nghiên cứu cho thấy rằng, mức giá mà hộ dân sẵn lòng trả trung bình là 6.200 đồng cho mỗi $\mathrm{m}^{3}$ nước sạch. Bên cạnh đó, chỉ có khoảng $32 \%$ hộ dân sã̃n lòng trả với mức giá 6.500 đồng hay cao hơn cho mỗi $\mathrm{m}^{3}$ nước sạch. Yếu tố thu nhập ảnh hưởng thuận chiều, trong khi quy mô nhân khẩu của hộ dân ảnh hưởng nghịch chiều đến mức sẵn lòng trả đối với mức giá 6.500 đồng hay cao hơn cho mỗi $\mathrm{m}^{3}$ nước sạch. Nghiên cứu này đề xuất nhà cung cấp xem xét gia tăng định mức lượng nước tối thiểu lên $10 \mathrm{~m}^{3}$ /tháng thay vì chỉ $4 \mathrm{~m}^{3}$ /tháng cho mỗi hộ gia đình tương ứng với mức giá tối thiểu 5.700 đồng cho mỗi $\mathrm{m}^{3}$ nước sạch sử dụng.

\section{1. Đặt Vấn Đề}

Trà Vinh là tỉnh ven biển thuộc đồng bằng sông Cửu Long (ĐBSCL), với dân số 1,04 triệu người (2016), trong đó dân số nông thôn chiếm 82,1\% dân số toàn tỉnh. Về khía cạnh sử dụng nước cho sinh hoạt trong quá trình xây dựng nông thôn mới cho thấy tỉnh đã có nhiều nổ lực để phục vụ người dân. Hiện toàn tỉnh có 225 trạm cấp nước có công suất từ $5-100 \mathrm{~m}^{3} /$ ngày, trong đó có 57 trạm có công suất từ $20-100 \mathrm{~m}^{3} /$ ngày, đã cung cấp cho hơn 66.645 hộ được sử dụng nước máy. Theo số liệu thống kê, có $86,20 \%$ dân số nông thôn được sử dụng nước hợp vệ sinh, trong đó $57,42 \%$ số hộ dân nông thôn được cấp nước đạt theo Quy chuẩn 02 của Bộ Y tế.

Trong 9 đơn vị hành chính của tỉnh, huyện Càng Long là một trong các đơn vị có số hộ sử dụng nước hợp vệ sinh thấp thứ 2 , hộ sử dụng nước sạch thấp thứ 6 , tổng số hộ sử dụng nước sạch là 145.422 hộ, chiếm 65,22\% (PCTV, 2017). Tuy nhiên, theo quy hoạch cung cấp nước sinh hoạt và vệ sinh môi trường nông thôn tỉnh Trà Vinh giai đoạn 2016 - 2020 và tầm nhìn đến năm 2030, dân cư nông thôn sử dụng nước sạch quy chuẩn chất lượng quốc gia đạt $75 \%$, với số lượng tối thiểu 80 lít/người/ngày, và theo Bộ tiêu chí quốc gia về nông thôn mới, đến 2020, tất cả dân cư nông thôn sử dụng nước sạch đạt tiêu chuẩn quốc gia (PCTV, 2018).

Trong năm 2016, tỉnh Trà Vinh đã thực hiện giá nước theo mô hình lũy tiến, hộ dân sử dụng nước càng nhiều trong một tháng thì giá nước trên mỗi đơn vị $\mathrm{m}^{3}$ càng tăng. Cụ thể, giá nước thấp nhất là 5.700 đồng mỗi m3 cho định mức nhỏ hơn $4 \mathrm{~m}^{3}$ /tháng, tăng dần lên 6.500 đồng, 7.300 đồng, 8.100 đồng và 9.000 đồng lần lượt cho các định mức tiêu thụ từ $4-10 \mathrm{~m}^{3} /$ tháng, $>10-15 \mathrm{~m}^{3}$ /tháng, > $15-20 \mathrm{~m}^{3}$ /tháng và trên $20 \mathrm{~m}^{3} /$ tháng (PCTV, 2016). Tìm kiếm các giải 
pháp nâng cao tỉ lệ hộ sử dụng nước sạch đạt tiêu chuẩn trên địa bàn của huyện được đặt ra như một yêu cầu cấp thiết. Gia tăng tỉ lệ sử dụng nước sạch trong nông thôn có thể đạt được từ gia tăng nguồn cung nước sạch từ công ty cấp nước trên địa bàn, đồng thời cũng phải cần có sự chấp nhận của người sử dụng nước về chất lượng nước được phục vụ, giá nước hợp lý, phù hợp với thu nhập của người dân và những yếu tố liên quan.

Nghiên cứu này có mục đích nhằm tìm hiểu mức sã̃n lòng trả (Willingness to pay: WTP) của hộ dân trong việc sử dụng nước sạch ở những khu vực nông thôn của huyện Càng Long, nơi có trạm cấp nước sạch nhưng các hộ dân chưa sử dụng. Kết quả nghiên cứu này giúp người dân và cơ quan liên quan ở địa phương có cách nhìn tổng thể về nguyện vọng của người dân trong sử dụng nước sạch, đồng thời giúp rút ra được những bài học cũng như điều chỉnh các biện pháp cung cấp nước sạch được hợp lý. Bài viết này có một phần nội dung được thể hiện trong luận văn thạc sĩ ngành phát triển nông thôn tại đại học Trà Vinh (Pham, 2019).

\section{Vật Liệu và Phương Pháp Nghiên Cứu}

\subsection{Vùng nghiên cứu}

Nghiên cứu này được thực hiện bằng cách tiếp cận điều tra xã hội học đối với hộ dân tại đia bàn huyện Càng Long, là huyện có tỉ lệ hộ chưa sử dụng nước sạch tương đối cao (khoảng 35\%) trong các huyện thị của tỉnh Trà Vinh. Huyện Càng Long có 18 trạm cấp nước tập trung do Trung tâm Nước sạch và Vệ sinh môi trường nông thôn trực thuộc Sở Nông nghiệp và PTNT tỉnh Trà Vinh quản lý có công suất từ $10-100 \mathrm{~m}^{3} /$ ngày.

Hai đơn vị xã Tân An và Huyền Hội có dân số lớn trong khi tỷ lệ sử dụng nước sạch lần lượt là $42,62 \%$ và $26,59 \%$, có thể xem là thấp nhất so với các xã và thị trấn của huyện, được chọn nghiên cứu. Đây là 2 xã có 2 trạm cấp nước sạch đóng trên địa bàn. Tuy nhiên, các xã này chưa đạt tiêu chí môi trường trong chuẩn xã nông thôn mới và trên tổng thể chỉ đạt từ 8 đến 9 tiêu chí trên 19 tiêu chí của bộ tiêu chí xã nông thôn mới (Bảng $1)$.

\subsection{Phương pháp thu thập số liệu và phân tích}

Phương pháp đánh giá ngẫu nhiên (Contigent Valuation Method: CVM) được trích dẫn trong David \& Petr (2010), Nguyen (2010) và Wattage
(2011), được áp dụng. Số liệu khảo sát thực hiện năm 2018 đối với 120 hộ dân được chọn theo thể thức phi xác suất, phân đều 60 hộ trên mỗi xã Tân An và Huyền Hội của huyện Càng Long, đây là những hộ ở cùng khu vực nơi có trạm cấp nước nhưng chưa sử dụng nước sạch, đồng thơi sẽ là những hộ có khả năng sẽ sử dụng nước sạch trong thời gian sắp tới. Nội dung thu thập ở hộ dân bao gồm các đặc điểm chính của gia đình và sự sẵn lòng trả của hộ dân với các mức giá khác nhau từ thấp đến cao. Đối với đặc điểm hộ dân, các thông tin được thu thập như đặc điểm của chủ hộ, nhân khẩu, diện tích đất đai canh tác, nghề nghiệp và thu nhập trong một tháng. Đối với câu hỏi sự sã̃n lòng trả, loại câu hỏi nhiều mức giá được đưa ra (bidding game) để hộ dân chọn lựa mức giá mà họ cho là phù hợp. Các mức giá trên mỗi $\mathrm{m}^{3}$ nước đưa ra trong phiếu phỏng vấn được tham chiếu từ Quyết định số 2024/QĐ-UBND ngày 26 tháng 9 năm 2016, gồm các mức từ 5.700, đến $6.000,6.300,6.500,7.000,7.300,8.000,8.100$ và 9.000 đồng.

Tỉ lệ số hộ dân sã̃n sàng chấp nhận trả với các mức giá khác nhau được thu thập, sắp xếp chúng theo thứ tự mức giá từ thấp đến cao. Tỉ lệ số hộ đồng ý với mức giá $\mathrm{T}_{\mathrm{j}}$ được tính từ tỉ lệ đồng $y$ ở mức giá $\mathrm{P}_{\mathrm{j}}$ trừ cho tỉ lệ ở mức giá cao hơn liền kề $\mathrm{P}_{\mathrm{j}+1}$ (Pham, 2010). Như vậy, WTP trung bình được tính từ tổng số tiền của tất cả các tỉ lệ số hộ đồng ý trả cho từng mức giá được khảo sát, được viết theo công thức dưới đây:

$$
\overline{\mathrm{WTP}}=\sum \mathrm{T}_{\mathrm{j}}\left(\mathrm{P}_{\mathrm{j}}-\mathrm{P}_{\mathrm{j}+1}\right)
$$

\section{Trong đó:}

WTP: WTP trung bình.

$\mathrm{T}_{\mathrm{j}}$ : Các mức giá.

$\mathrm{P}_{\mathrm{j}}$ : Tỉ trọng số hộ đồng ý với các mức giá trong tổng số hộ khảo sát.

Bên cạnh nội dung trên đây, nghiên cứu này còn xem xét các yếu tố ảnh hưởng đến sự sẵn lòng trả của hộ dân đối với mức giá 6.500 đồng, là mức giá được áp dụng cho định mức từ 4 - 10 $\mathrm{m}^{3} /$ hộ/tháng vốn là định mức rất phổ biến ở hộ gia đình nông thôn. Để xem xét sự sã̃n lòng trả này, hàm hồi quy được thiết lập có dạng như sau:

$$
\mathrm{Y}=\alpha+\beta_{\mathrm{i}} \mathrm{X}_{\mathrm{i}}+\varepsilon
$$

Trong đó:

Y: Nhận giá trị 1 nếu hộ dân đồng ý trả với mức giá bằng hoặc thấp hơn 6.500 đồng, và 0 nếu hộ dân trả với mức giá cao hơn 6.500 dồng. 
Bảng 1. Đặc điểm các xã khảo sát theo tiêu chí sử dụng nước sạch

\begin{tabular}{lccc}
\hline Các tiêu chí & Xã Tân An & Xã Huyền Hội & Huyện Càng Long \\
\hline Dân số (người) & 10.468 & 14.224 & 171.955 \\
Tỷ lệ sử dụng nước sạch (\%) & 42,62 & 26,59 & 65,22 \\
Tỷ lệ sử dụng nước hợp vệ sinh $(\%)$ & 99,32 & 54,50 & 73,67 \\
Số lượng trạm cấp nước (trạm) & 01 & 01 & 15 \\
Số tiêu chí đạt xã NTM 08/19 & $09 / 19$ & - & \\
Tiêu chí môi trường trong xã NTM & Chưa đạt & Chưa đạt & - \\
\hline Nguồn: PCTV, 2017. & & &
\end{tabular}

$\alpha$ : Hệ số xác định.

$\beta_{\mathrm{i}}$ : Hệ số biên của các yếu tố giải thích.

$\mathrm{X}_{\mathrm{i}}$ : Các biến giải thích.

Vì các giá trị của Y có 2 trạng thái là 0 và 1 có tính xác suất, nên nếu gọi p là xác suất để biến cố hộ dân đồng ý với mức giá bằng hay cao hơn 6.500 đồng $/ \mathrm{m}^{3}$ thì $1-\mathrm{p}$ là xác suất để biến cố không đồng ý xảy ra. Phương trình hồi qui trên được chuyển thành mô hình hồi quy binary logistic có dạng như sau:

$$
\ln \left(\frac{\mathrm{p}}{1-\mathrm{p}}\right)=\alpha+\beta_{\mathrm{i}} \mathrm{X}_{\mathrm{i}}+\varepsilon
$$

Từ (3), xác suất p xảy ra được ước lượng dựa vào các hệ số ước lượng $\alpha, \beta_{\mathrm{i}}$ và giá trị của $\mathrm{X}_{\mathrm{i}}$ như dưới đây:

$$
\mathrm{p}=\frac{\mathrm{e}^{\alpha+\beta_{\mathrm{i}} X_{\mathrm{i}}}}{1+\mathrm{e}^{\alpha+\beta_{\mathrm{i}} X_{\mathrm{i}}}}
$$

Các hệ số hồi quy $\beta_{\mathrm{i}}$ được uớc luợng bằng phương pháp hợp lý cực đại (Maximum Livelihood). Đại lượng Wald Chi-Square được sử dụng để kiểm định ý nghĩa thống kê của hệ số hồi quy tổng thể. Cách thức sử dụng mức ý nghĩa (Sig) cho kiểm định Wald cũng theo quy tắc nếu Sig nhỏ hơn 0,05 thì bác bỏ $\mathrm{H}_{0}\left(\beta_{\mathrm{i}}=0\right)$, tức hệ số có ý nghĩa thống kê.

Tổ hợp liên hệ tuyến tính của toàn bộ các hệ số trong mô hình cũng được kiểm định mức ý nghĩa trong việc giải thích cho biến phụ thuộc, trong đó kiểm định Chi-Square được sử dụng, nếu Sig nhỏ hơn 0,05 thì bác bỏ $\mathrm{H}_{0}$, tức mô hình có độ phù hợp tốt, ngược lại, nếu Sig lớn hơn 0,05 thì chấp nhận giả thuyết $\mathrm{H}_{0}$ (các hệ số hồi quy đều bằng 0 : $\beta_{1}=\beta_{2}=\ldots=\beta_{\mathrm{k}}=0$ ). Độ phù hợp tổng quát cũng có thể được đánh giá dựa trên chỉ tiêu -2LL (-2 Log Likelihood), giá trị -2LL càng nhỏ càng thể hiện độ phù hợp cao. Giá trị nhỏ nhất của -2LL là 0 (tức là không có sai số) khi đó mô hình có một độ phù hợp hoàn hảo.

\section{Kết Quả và Thảo Luận}

Hộ gia đình khu vực nông thôn ở 2 xã khảo sát có đặc điểm như trình bày ở Bảng 1 . Trong đó độ tuổi của chủ hộ tương đối trẻ, trung bình (TB) khoảng 41,7 tuổi và có độ lệch chuẩn (ĐLC) thấp so tuổi trung bình. Quy mô nhân khẩu của hộ cũng rất tương đồng với quy mô hộ của khu vực nông thôn nói chung, chỉ khoảng 4,1 người/hộ. Nguyên nhân do ở khu vực này mỗi xã chỉ có 1 trạm cấp nước nên khoảng cách từ hộ khảo sát đến trạm cấp nước tương đối xa, trung bình là $2,3 \mathrm{~km}$ và cũng khá biến động với ĐLC là $1,4 \mathrm{~km}$ (Bảng 2). Mặt khác, nhân khẩu hộ gia đình nhỏ trong khi nghề nghiệp của chủ hộ ở Bảng 3 cho thấy tỉ trọng hộ có nghề nghiệp phi nông nghiệp tương đối cao, chiếm 46,7\% nên nhìn chung thu nhập của hộ gia đình đạt 9,9 triệu đồng/hộ/tháng. Đây là mức thu nhập tương đối khi so với thu nhập nói chung của khu vực nông thôn vốn bị lệ thuộc nhiều vào sản xuất nông nghiệp.

Mặc dù ở nông thôn nhưng nhờ nghề nghiệp của hộ dân ở khu vực khảo sát có tỉ lệ làm nông nghiệp không cao, chỉ chiếm khoảng 20,8\% đối với chủ hộ được khảo sát, phần còn lại là làm thuê trong khu vực nông nghiệp chiếm 32,5\%. Điều này có lẽ do giao thông thuận lợi, cũng như do vị trí địa lý ở gần khu vực thị trấn huyện nên tỉ lệ chủ hộ có tham gia làm phi nông nghiệp khá cao chiếm 46,7\%. Trong đó, viên chức nhà nước chiếm đến $16,7 \%$ và kinh doanh phi nông nghiệp chiếm đến $30 \%$ số chủ hộ khảo sát (Bảng 3). Các yếu tố nghề nghiệp này sẽ được xem xét đến khả năng chấp nhận các mức giá sử dụng nước ở phần sau của bài viết này.

Hiện các hộ dân đang sử dụng nước từ nhiều nguồn khác nhau và chưa sử dụng nước sạch từ hệ thống các trạm cấp nước ở trong xã. Nhận thức của hộ dân về sử dụng nước sạch vì vậy khá rõ ràng và được thể hiện ở Hình 1 . Các hộ dân 
Bảng 2. Đặc điểm hộ gia đình liên quan đến sử dụng nước sạch theo xã

\begin{tabular}{lcccccc}
\hline \multirow{2}{*}{ Đặc điểm } & \multicolumn{2}{c}{ Xã Tân An } & \multicolumn{2}{c}{ Xã Huyền Hội } & \multicolumn{2}{c}{ Tổng cộng } \\
\cline { 2 - 7 } & TB & ĐLC & TB & ĐLC & TB & ĐLC \\
\hline Tuổi (năm) & 43,0 & 12,5 & 40,3 & 10,5 & 41,7 & 11,5 \\
Nhân khẩu (người/hộ) & 4,5 & 1,3 & 3,8 & 0,9 & 4,1 & 1,2 \\
Nhà cách trạm (km) & 2,1 & 1,5 & 2,4 & 1,3 & 2,3 & 1,4 \\
Thu nhập (triệu đồng/tháng) & 10,5 & 5,5 & 9,4 & 3,7 & 9,9 & 4,7 \\
\hline
\end{tabular}

TB: Trung bình, ĐLC: Độ lệch chuẩn.

Bảng 3. Đặc điểm nghề nghiệp của hộ dân theo xã

\begin{tabular}{ccccccc}
\hline \multirow{2}{*}{ Đặc điểm } & \multicolumn{2}{c}{ Xã Tân An } & \multicolumn{2}{c}{ Xã Huyền Hội } & \multicolumn{2}{c}{ Tồng cộng } \\
\cline { 2 - 7 } & Tần số & $(\%)$ & Tần sốn & $(\%)$ & Tần số & $(\%)$ \\
\hline Phi Nông nghiệp & 27 & 45,0 & 29 & 48,4 & 56 & 46,7 \\
Kinh doanh & 20 & 33,3 & 16 & 26,7 & 36 & 30,0 \\
Viên chức & 7 & 11,7 & 13 & 21,7 & 20 & 16,7 \\
Nông nghiệp & 33 & 55,0 & 31 & 51,6 & 54 & 53,3 \\
Nông dân & 13 & 21,7 & 12 & 20,0 & 25 & 20,8 \\
Làm thuê & 20 & 33,3 & 19 & 31,7 & 39 & 32,5 \\
Tồng cộng & 60 & 100,0 & 60 & 100,0 & 120 & 100,0 \\
\hline
\end{tabular}

cho rằng nguồn nước sông mà họ đang sử dụng là không đủ cung cấp trong một số thời điểm ở mùa khô, đồng thời bị ô nhiễm do nhiều nguồn ô nhiễm khác nhau. Ngoài ra, các hộ dân cũng nhận thấy rằng nhu cầu sử dụng nước sạch là rất cần thiết, giúp cho điều kiện vệ sinh và sinh hoạt được tốt hơn. Đối với nhiều hộ dân làm kinh doanh hay viên chức nhà nước, việc sử dụng nước sạch từ hệ thống cấp nước cũng giúp tiết kiệm được thời gian trong việc lấy và xử lý nước so với điều kiện hiện tại.

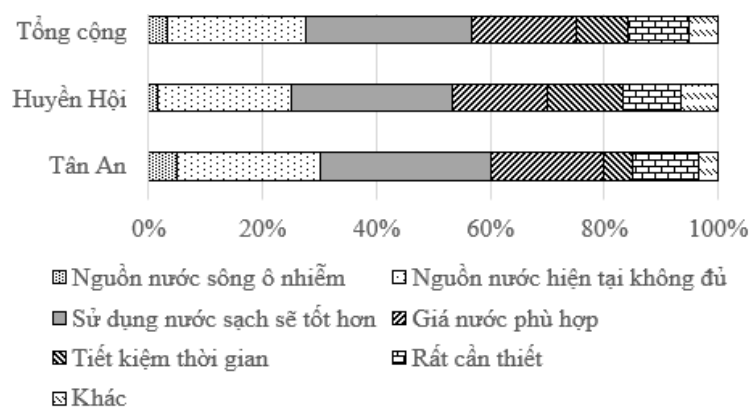

Hình 1. Các nguyên nhân chấp nhận sử dụng nước sạch theo xã.

Từ những đặc điểm hộ gia đình cũng như nghề nghiệp và nhận thức của người dân như đề cập ở trên, tình trạng hộ dân bằng lòng trả với các mức giá nước sạch được khảo sát khi giả định họ sẽ sử dụng. Các mức giá đưa ra khảo sát được tham chiếu từ QĐ 2024/QĐ-UBND ngày 26 tháng 9 năm 2016 của UBND tỉnh Trà Vinh. Kết quả cho thấy rằng có khoảng 37,5\% số hộ đồng ý với mức giá cao nhất là 6.500 đồng. Tuy nhiên, nếu mức giá thấp hơn thì tỉ lệ số hộ đồng ý sẽ cao hơn. Cụ thể, với mức giá 6.000 đồng thì số hộ đồng ý sẽ là $55 \%, 5.700$ đồng thì tỉ lệ này sẽ là $85 \%$ và nếu 5.000 đồng thì sẽ có $100 \%$ số hộ đồng ý sẵn lòng trả. Với các mức giá cao hơn từ 7.000 đồng đến 9.000 đồng cho mỗi m3 thì tỉ lệ số hộ sãn lòng trả sẽ thấp hơn (Bảng 4). Các tỉ lệ số hộ chấp nhận với các mức giá này khá phù hợp với nghiên cứu của Nguyen (2017) tại Hà Nội.

Từ số liệu của Bảng 3 , để ước tính mức sẵn lòng trung bình mà các hộ dân sẵn lòng trả theo công thức (1) được xác định như sau:

$\overline{\mathrm{WTP}}=5000 \times(100-85)+5700 \times(85-55)$ $+6000 \times(55-38,3)+6300 \times(38,3-37,5)+$ $6500 \times(37,5-25,8)+7000 \times(25,8-15)+7300$ $\times(15-9,2)+8000 \times(9,2-4,2)+8100 \times(4,2$ $-0,8)+9000 \times(0,8-0)=6200$ (đồng).

Nhằm xem xét mức sẵn lòng trả bằng và cao hơn 6.500 đồng của hộ dân chịu tác động bởi yếu tố nào, một hàm hồi quy nhị phân binary logistic được thực hiện và kết quả được trình bày ở Bảng 5.

Với kết quả ước lượng cho thấy hàm số có mức ý nghĩa Sig $=0,007$ nhỏ hơn so với mức $\alpha=0,05$ nên giả thuyết H0 bị bác bỏ, hay nói cách khác hàm số có ý nghĩa thống kê. Bên cạnh đó, mức ý nghĩa của hệ số ước lượng 2 yếu tố nhân khẩu 
Bảng 4. Tỉ lệ hộ dân đáp ứng với các mức giá nước tại điểm khảo sát

\begin{tabular}{|c|c|c|c|c|c|c|}
\hline $\begin{array}{l}\text { Thứ tự } \\
\text { mức giá } \\
\text { (j) }\end{array}$ & $\begin{array}{c}\text { Mức giá } \\
\text { (đồng/m³) } \\
\left(\mathrm{T}_{\mathrm{j}}\right)\end{array}$ & $\begin{array}{l}\text { Số hộ } \\
\text { khảo sát } \\
\text { (n) }\end{array}$ & $\begin{array}{c}\text { Số hộ } \\
\text { đồng ý } \\
\text { mức giá j }\end{array}$ & $\begin{array}{l}\text { Tỉ lệ \% hộ } \\
\text { dồng ý mức } \\
\text { giá j trên số } \\
\text { hộ khảo sát } \\
\quad\left(\mathrm{P}_{\mathrm{j}}\right)\end{array}$ & $\begin{array}{l}\text { Số hộ cộng } \\
\text { dồn đồng ý } \\
\text { mức giá j } \\
\left(Y_{\mathrm{j}}\right)\end{array}$ & $\begin{array}{c}\text { Tỉ lệ \% cộng } \\
\text { dồn đồng ý } \\
\text { mức giá } \mathrm{j} \\
\left(\mathrm{Y}_{\mathrm{j}} / \mathrm{n}\right)\end{array}$ \\
\hline 1 & 5000 & 120 & 18 & 15,0 & 120 & 100,0 \\
\hline 2 & 5700 & 120 & 36 & 30,0 & 102 & 85,0 \\
\hline 3 & 6000 & 120 & 20 & 16,7 & 66 & 55,0 \\
\hline 4 & 6300 & 120 & 1 & 0,8 & 46 & 38,3 \\
\hline 5 & 6500 & 120 & 14 & 11,7 & 45 & 37,5 \\
\hline 6 & 7000 & 120 & 13 & 10,8 & 31 & 25,8 \\
\hline 7 & 7300 & 120 & 7 & 5,8 & 18 & 15,0 \\
\hline 8 & 8000 & 120 & 6 & 5,0 & 11 & 9,2 \\
\hline 9 & 8100 & 120 & 4 & 3,3 & 5 & 4,2 \\
\hline \multirow[t]{2}{*}{10} & 9000 & 120 & 1 & 0,8 & 1 & 0,8 \\
\hline & Tổng cộng & 120 & 120 & 100,0 & 120 & 100,0 \\
\hline
\end{tabular}

Bảng 5. Kết quả ước lượng các yếu tố ảnh hưởng đến sự sẵn lòng trả với mức giá bằng và cao hơn 6.500 đồng

\begin{tabular}{|c|c|c|c|c|c|c|}
\hline Các biến giải thích & B & S.E. & Wald & $\mathrm{df}$ & Sig. & $\operatorname{Exp}(B)$ \\
\hline Tuổi (năm) & 0,053 & 0,023 & 5,481 & 1 & $\overline{0,019}$ & 1,055 \\
\hline Giới tính $(0,1)^{(\mathrm{a})}$ & 0,745 & 0,451 & 2,732 & 1 & 0,098 & 2,106 \\
\hline Trình độ $(0,1)^{(\mathrm{b})}$ & 0,411 & 0,464 & 0,784 & 1 & 0,376 & 1,508 \\
\hline Nghề nghiệp $(0,1)^{(\mathrm{c})}$ & $-0,041$ & 0,419 & 0,009 & 1 & 0,923 & 0,960 \\
\hline Nhân & $-0,554$ & 0,226 & 6,023 & 1 & 0,014 & 0,574 \\
\hline âp (triệu đồng) & 0,152 & 0,055 & 7,582 & 1 & 0,006 & 1,164 \\
\hline ạm $(\mathrm{km})$ & $-0,261$ & 0,159 & 2,689 & 1 & 0,101 & 0,771 \\
\hline Hằng số & $-2,128$ & 1,195 & 3,169 & 1 & 0,075 & 0,119 \\
\hline
\end{tabular}

- 2 Log livelihood = 140,611; Cox \& Snell R = 0,140;

Nagelkerke R square $=0,191$; Sig. $=0,007 ; \mathrm{N}=120$

(a) Giới tính: $0=$ nữ; $1=$ nam).

(b) Trình độ ( $0=$ học vấn từ THCS trở xuống; 1 = học vấn từ THPT trở lên).

(c) Nghề nghiệp ( $0=$ nông nghiệp; $1=$ phi nông nghiệp).

và thu nhập có giá trị < 0,05 nên các hệ số ước lượng này có ý nghĩa thống kê, trong khi các yếu tố khác được nêu trong Bảng 5 đều có giá trị Sig lớn hơn 0,05 nên không có ý nghĩa thống kê. Như vậy, hàm số (3) được viết với các kết quả của hệ số ước lượng như sau:

$\ln \left(\frac{\mathrm{p}}{1-\mathrm{p}}\right)=-0,554 \times$ Nhankhau $+0,152 \times$ Thunhap

Từ kết quả trên cho thấy rằng, khả năng chấp nhận giá nước ở mức giá bằng hay cao hơn 6.500 đồng chỉ chịu tác động bởi 2 yếu tố quy mô nhân khẩu và thu nhập của hộ gia đình, trong đó quy mô nhân khẩu tác động nghịch chiều và thu nhập tác động thuận chiều với khả năng chấp nhận mức giá từ bằng đến cao hơn 6.500 đồng. Thu nhập càng cao càng có khả năng chấp nhận mức giá này càng cao, trong khi số nhân khẩu của hộ gia đình càng cao thì khả năng chấp nhận giá này càng thấp vì lượng nước sử dụng của hộ gia đình sẽ tăng lên khi số nhân khẩu gia tăng trong khi giá nước không được thiết lập ở mức tối thiểu 5.700 đồng $/ \mathrm{m}^{3}$ như trong QĐ2024/QĐ-UBND. Để cụ thể hóa xác suất chấp nhận bằng hay cao hơn mức giá này, hàm xác suất (4) được ước lượng và thay thế giá trị trung bình của nhân khẩu, thu 
nhập của hộ gia đình ở Bảng 1 và với giá trị e $=$ 2,7182 vào (4) ta có xác suất p như sau:

$$
\begin{aligned}
\mathrm{p} & =\frac{\mathrm{e}^{\alpha+\beta_{\mathrm{i}} X_{\mathrm{i}}}}{1+\mathrm{e}^{\alpha+\beta_{\mathrm{i}} X_{\mathrm{i}}}}=\frac{\mathrm{e}^{-0,554 \times 4,1+0,152 \times 9,9}}{1+\mathrm{e}^{-0,554 \times 4,1+0,152 \times 9,9}} \\
& =0,32=32 \%
\end{aligned}
$$

Xác suất $\mathrm{p}$ nói trên cho thấy rằng, khi yếu tố thu nhập không thay đổi, nếu quy mô nhân khẩu tăng lên 1 người (5,1 người/hộ) so với giá trị trung bình như hiện tại $(4,1$ người/hộ), xác suất $\mathrm{p}$ sẽ giảm còn $21 \%$, tương tự như thế nếu nhân khẩu tăng lên thành 6,1 và 7,1 người/hộ thì xác suất chấp nhận $\mathrm{p}$ giảm xuống còn $13 \%$ và $8 \%$ tương ứng. Điều này có nghĩa nếu định mức từ 4 - 10 $\mathrm{m}^{3}$ /hộ/tháng cho gia đình đông nhân khẩu trên 5 người trở lên, nếu sử dụng mức giá bằng hay cao hơn 6.500 đồng thì khả năng chấp nhận của người dân sẽ thấp xuống, nói cách khác, nếu quy mô nhân khẩu 5,1 người/hộ đến 6,1 người/hộ thì nhu cầu nước tối thiểu của họ trong tháng sẽ cao hơn định mức này, đồng thời mức giá tối thiểu cần áp dụng tương ứng.

\section{Kết Luận và Kiến Nghị}

\subsection{Kết luận}

Hộ gia đình nông thôn ở địa điểm khảo sát có những đặc điểm quy mô nhân khẩu, thu nhập khá tương đồng với khu vực nông thôn nói chung. Người dân có những nhận thức tốt, hiểu được tầm quan trọng của việc sử dụng nước sạch trong đời sống gia đình và họ có nhu cầu sử dụng nước sạch khá cao.

Trung bình mức giá sẵn lòng chấp nhận cho 1 $\mathrm{m}^{3}$ là 6.200 đồng. Tuy nhiên, nếu mức giá thấp hơn thì số tỉ lệ hộ sẵn lòng chấp nhận cao hơn. Các yếu tố quan trọng tác động thuận chiều với sự chấp nhận giá nước bằng hay cao hơn 6.500 đồng là thu nhập của hộ gia đình, trong khi yếu tố tác động nghịch chiều với sự bằng lòng này là quy mô nhân khẩu của hộ gia đình. Trung bình có khoảng $32 \%$ số hộ chấp nhận bằng hay cao hơn mức giá này. Càng tăng quy mô nhân khẩu trong khi các yếu tố khác không đổi thì khả năng chấp nhận mức giá này càng thấp.

\subsection{Kiến nghị}

Công tác cung cấp dịch vụ nước sạch cho khu vực nông thôn đang là nhu cầu thiết thực của người dân, vì vậy, chính quyền địa phương và các công ty cấp nước cần xem xét các khả năng để tăng cường quy mô cấp nước cho khu vực nông thôn. Bên cạnh đó, cần xem xét gia tăng định mức sử dụng nước tối thiểu lên $10 \mathrm{~m}^{3}$ /tháng/hộ thay vì chỉ $4 \mathrm{~m}^{3}$ /tháng/hộ cùng với mức giá tối thiểu tương ứng là 5.700 đồng cho mỗi $\mathrm{m}^{3}$ để gia tăng khả năng chấp nhận sử dụng nước của cộng đồng dân cư.

\section{Tài Liệu Tham Khảo (References)}

David, H., \& Petr, M. (2010). Contingent valuation: past, present and future. Prague Economic Paper 19(4), 329-343.

Nguyen, H. B. (2017). Estimation of local resident's willingness to pay for using clean water in Chuong My district, Ha Noi. Journal of Forestry Science and Technology 2, 129-139.

Nguyen, S. V. (2010). Methods for evaluating the value of natural resources and environment. Ha Noi, Vietnam: Hanoi University of Agriculture.

PCTV (People's Committee of Tra Vinh province). (2018). Review, adjustment and planning supplement of water for daily life and rural environmental sannitation for the period of 2016-2020 and vision to 2030 in Tra Vinh province. Tra Vinh, Vietnam: PCTV Office.

PCTV (People's Committee of Tra Vinh province). (2017). A report on monitoring and evaluation of clean water and rural environmental sannitation in 2016. Tra Vinh, Vietnam: PCTV Office.

PCTV (People's Committee of Tra Vinh province). (2016). Decision No. 2024/QD-UBND dated 26/9/2016. Tra Vinh, Vietnam: PCTV Office.

Pham, M. H. (2010). Financing environmental protection activities in Nha Trang Bay: The role of the visitors. Journal of Fisheries Science and Technology 1, 79-87.

Pham, T. D. (2019). Evaluation of the current status of supply and demand for clean water of stakeholders in Cang Long district, Tra Vinh province. (Unpublished master's thesis). School of Agri-Aquaculture, Tra Vinh University.

Wattage, P. (2011). A targeted literature review - contingent valuation method. Retrieved from October 1, 2018, http://citeseerx.ist.psu.edu/doi=0.1.1.195.1300. 